\title{
Chronic oestrogen replacement in ovariectomised rats attenuates food intake and augments c-Fos expression in the suprachiasmatic nucleus specifically during the light phase
}

\author{
Akira Takamata*, Kayo Toriit, Kana Miyake and Keiko Morimoto \\ Department of Environmental Health, Nara Women's University, Kitauoya Nishimachi, Nara 630-8506, Japan \\ (Received 27 September 2010 - Revised 28 February 2011 - Accepted 2 March 2011 - First published online 31 May 2011)
}

\section{Abstract}

Oestrogen replacement in ovariectomised (OVX) rats has been reported to attenuate food intake, especially during the light phase. To gain better insight into the central mechanism of oestrogen-induced reduction of food intake, we examined the effect of chronic oestrogen replacement in OVX rats on c-Fos expression in the suprachiasmatic nucleus (SCN) and on food intake during the light and dark phases. Eight-week-old female rats were ovariectomised and implanted with either an oestradiol (E2) or a vehicle pellet (Veh) subcutaneously. The animals were housed in an environment with a $12 \mathrm{~h}$ light $-12 \mathrm{~h}$ dark cycle with the lights on at 07.00 hours. The amount of spontaneous food intake relative to each animal's body weight was significantly less for the E2 group than for the Veh group during the light phase, but there were no differences shown between these groups during the dark phase. There were no differences shown in the number of c-Fos-immunoreactive cells in the SCN in the E2 group compared with the Veh group during the early dark phase (22.00 hours; Zeitgeber time 15.00 (ZT15)), but the number was significantly higher than in the Veh group during the early light phase (10.00 hours; ZT3). This finding suggests that chronic oestrogen replacement chronically enhances SCN activity, specifically during the light phase. The oestrogen-induced enhancement of SCN activity during the light phase is possibly involved in the light phase-specific attenuation of food intake by oestrogen replacement in OVX rats.

Key words: Oestrogen: Food intake: Suprachiasmatic nucleus: Diurnal rhythm: Ovariectomy

It is well established that female gonadal steroids play an important role in regulating body weight, body composition and food intake ${ }^{(1-4)}$. An ovariectomy (OVX) in rats increases food intake and body weight, and chronic oestrogen replacement reverses these increases, suggesting that an oestrogen, but not a progesterone, deficit increases food intake and body weight ${ }^{(5-7)}$

Food intake patterns show significant circadian variation, and intact rats eat about $80 \%$ of their daily intake during the dark phase, and rats start and terminate eating immediately after phase change ${ }^{(7-10)}$. Varma et al. ${ }^{(7)}$ reported that chronic oestrogen replacement tonically decreases food intake, mainly during the light phase. Thus, the anorexigenic effect of oestrogen replacement might be specific to the light phase. However, the central mechanism for the light phase-specific attenuation of food intake that is induced by oestrogen remains unknown.

The hypothalamic suprachiasmatic nucleus (SCN) is the main oscillator that generates the circadian rhythm of behaviours $^{(11-15)}$, and bilateral lesions of the SCN abolish the daily oscillation of food intake ${ }^{(10)}$, suggesting that the efferent signals from the SCN play a critical role in generating the daily variation in food intake. It has also been reported that the number of c-Fos immunoreactive (c-Fos-IR) cells in the SCN is more during the light phase than during the dark phase, suggesting that the SCN is more active during the light phase $\mathrm{e}^{(16-20)}$. Moreover, it is now clear that disruption of the circadian rhythm by clock gene deletion results in obesity ${ }^{(15,21,22)}$. Thus, the increase in SCN activity during the light phase might be associated with the decrease in food intake during this phase. In addition, oestrogen receptors $\alpha$ and $\beta$ are expressed in the $\mathrm{SCN}^{(23)}$ and that $17 \beta$-oestradiol modifies neuronal cell excitability and neurotransmission in the SCN of rats in vitro ${ }^{(24)}$.

Considering these findings together, we hypothesised that oestrogen increases SCN neuronal activity specifically during the light phase, which causes the oestrogen-induced reduction in food intake during this phase.

Abbreviations: c-Fos-IR, c-Fos immunoreactive; dm-SCN, dorsomedial SCN; E2, oestradiol; i-SCN, intermediary SCN; Veh, vehicle; SCN, suprachiasmatic nucleus; vl-SCN, ventrolateral SCN; ZT, Zeitgeber time.

*Corresponding author: Dr A. Takamata, fax +81 74220 3469, email takamata@cc.nara-wu.ac.jp

†Present address: Health Care R\&D Division, Sunstar, Inc., Osaka, Japan 
Fatehi \& Fatehi-Hassanabad ${ }^{(24)}$ reported that oestrogen increased neuronal activity in the SCN using slice preparation. However, they did not examine the effect during the light and dark phases, and the SCN was isolated from the input signals from other brain regions. Several studies have also demonstrated that acute oestrogen administration modifies c-Fos expression in the $\mathrm{SCN}$ in relation to the regulation of the luteinising hormone (LH) surge ${ }^{(25,26)}$. Peterfi et al. ${ }^{(20)}$ also demonstrated that c-Fos expression in the SCN in OVX rats with acute oestrogen replacement was less than in OVX rats without replacement during the light phase ( $8 \mathrm{~h}$ after light onset), but was not during the dark phase ( $8 \mathrm{~h}$ after dark onset). However, those studies examined the effect of acute oestrogen replacement, in which rats received a single injection of oestradiol $^{(20,26)}$ or implanted an oestradiol-containing capsule ${ }^{(25)}$ 1 or $2 \mathrm{~d}$ before the examination of $\mathrm{c}$-Fos expression. In these studies, the acute transient change in systemic oestradiol concentration might be a factor for the oestradiol-induced modification of neuronal activity or c-Fos expression in the SCN. It is still unknown whether chronic oestradiol replacement tonically affects the diurnal SCN activity rhythm. It is necessary to examine the effect of chronic oestrogen replacement on the diurnal variation of SCN activity and its relationship with spontaneous food intake to elucidate the mechanism for the oestrogen-induced tonic inhibition of food intake during the light phase.

In the present study, to gain better insight into the central mechanisms of the oestrogen-induced reduction of food intake, we examined the effect of chronic oestrogen replacement on the c-Fos expression in the SCN and on spontaneous food intake during the early light and early dark phases. We performed an examination of c-Fos expression in the SCN during the early light phase (at Zeitgeber time 3.00 (ZT3)) and early dark phase (ZT15) because rats start and terminate eating immediately after phase change ${ }^{(9)}$.

\section{Material and methods}

All experiments were conducted in accordance with the institutional guidelines for animal care, and all experimental procedures were approved by the Animal Care and Use Committee at Nara Women's University.

Eight-week-old female Wistar rats weighing 179.2 (SEM $2 \cdot 1) \mathrm{g}$ ( $n$ 28) were randomly assigned to two groups: the oestrogen-replaced (E2; $n$ 14) group and the vehicle-replaced (Veh; $n$ 14) group. Under pentobarbital anaesthesia (Nembutal; $40-45 \mathrm{mg} / \mathrm{kg}$; intraperitoneal), the rats were bilaterally ovariectomised and implanted subcutaneously with an oestradiol pellet (E2 group; $17 \beta$-oestradiol, $0.5 \mathrm{mg}$ /pellet, $21 \mathrm{~d}$ release; Innovative Research of America, Sarasota, FL, USA) or a vehicle pellet (Veh group; Innovative Research of America) through the interscapular incision on the same day. All of the rats were housed in a chamber with a controlled ambient temperature of $24^{\circ} \mathrm{C}$, a relative humidity of $40 \%$ and a $12 \mathrm{~h}$ light $-12 \mathrm{~h}$ light cycle with the lights on at $07.00 \mathrm{~h}$, and allowed access to standard rodent chow (CE-2; CLEA, Tokyo, Japan) and tap water ad libitum.
Following a $6 \mathrm{~d}$ recovery period after surgery, we measured spontaneous food intake every $12 \mathrm{~h}$ at 07.00 hours (ZT0) and 19.00 hours (ZT12) for a week in twelve out of twenty-eight rats ( $n 6$ for both the E2 and the Veh groups). Food intake was measured by manually weighing chow hoppers on a digital scale and corrected by subtracting the weight of food spillage. The mean food intake during the light and dark phases over these $7 \mathrm{~d}$ for six rats in both the E2 and the Veh groups was used for further analysis.

On the 14th day after surgery, the rats were killed for an immunohistochemical examination of the c-Fos expression in the SCN during the early light and dark phases ( $n 7$ in each group in each phase). Four rats in each group in each phase, in which food intake was not measured, were kept under exactly the same conditions as those for the rats in which food intake was measured. Data for the c-Fos expression in the SCN from one rat in the Veh group was not obtained during the light phase $(n 6)$ due to technical trouble.

The rats were deeply anaesthetised with pentobarbital at 10.00 hours (ZT3) or 22.00 hours (ZT15); blood samples were collected by cardiac puncture and the rats were transcardially perfused with ice-cold PBS, followed by $4 \%$ paraformaldehyde in a $0 \cdot 1 \mathrm{~m}$-phosphate buffer $(\mathrm{pH} 7 \cdot 4)$ for fixation. The brain was removed and immersed in the fixative for $2 \mathrm{~d}$ at $4{ }^{\circ} \mathrm{C}$. The fixed brain was immersed in $25 \%$ sucrose in PBS for $2 \mathrm{~d}$ at $4^{\circ} \mathrm{C}$ for cryoprotection. Frozen sections were cut at a thickness of $40 \mu \mathrm{m}$ with a freezing microtome (FX-801; Yamato kohki, Asaka, Japan). Free-floating sections were pretreated with $0 \cdot 1 \% \mathrm{H}_{2} \mathrm{O}_{2}$ in PBS for $20 \mathrm{~min}$ and then treated with $5 \%$ normal goat serum in PBS containing $0.3 \%$ Triton X-100 for $2 \mathrm{~h}$ at room temperature. Then, the sections were incubated with polyclonal anti-c-Fos antibody (Sc-52; Santa Cruz Biotechnology, Santa Cruz, CA, USA) diluted at 1:4000 in PBS containing $0.3 \%$ Triton X-100 and $1 \%$ normal goat serum for $16 \mathrm{~h}$ at $4^{\circ} \mathrm{C}$. After washing with PBS three times, the sections were incubated with biotinylated goat anti-rabbit IgG (dilution 1:400, BA-100; Vector, Burlingame, CA, USA) for $2 \mathrm{~h}$, followed by the ABC Elite kit solution (dilution 1:400; Vector) for $2 \mathrm{~h}$. Visualisation of the antibodies was performed with $0.02 \%$ 3,3-diaminobenzidine and $0.01 \% \mathrm{H}_{2} \mathrm{O}_{2}$ in a $50 \mathrm{~mm}$-Tris- $\mathrm{HCl}$ buffer ( $\mathrm{pH} 7.4)$. Sections were mounted on gelatin-coated glass slides, dehydrated with graded ethanol and cleared with Lemosol ${ }^{\circledR}$ and coverslipped.

Nine consecutive sections containing the SCN (in between -0.60 to $-0.96 \mathrm{~mm}$ from the bregma) were observed using a microscope (Olympus BX-50, Tokyo, Japan), and images were obtained using a cooled charge-coupled device (CCD) camera (Micropublisher RTV 5.0; QImaging, Surrey, BC, Canada). The SCN region was identified using the rat brain stereotaxic atlas ${ }^{(27)}$, and sections were carefully matched across all animals in all experimental groups.

The number of c-Fos-IR cells in the SCN as a whole and in the ventrolateral (vl-SCN), dorsomedial (dm-SCN) and intermediary (i-SCN) subregions of the unilateral SCN was examined because the neurotransmitters contained in these subdivisions and functions of the subdivisions were different for each area (see details in the discussion section). 
The ventrolateral region can be easily identified in the sections of the light phase, and we designated this portion as vl-SCN. There is another area in which c-Fos was densely expressed in the dorsal region along the third ventricle in the early light phase. We divided the dorsal portion further into the medial (dm-SCN) and intermediary part (i-SCN). Our preliminary experiment showed that the vl-SCN region matched well with the distribution of the vasoactive intestinal peptide neurons in the SCN. The number of c-Fos-IR cells in the whole and subregional SCN was counted for each of the nine consecutive sections using image analysis software (ImageJ; $\mathrm{NIH}$, Bethesda, MD, USA), and the sum of the number of c-Fos-IR cells for the nine sections in each rat was used for further analysis.

Blood samples were collected into the chilled EDTA-treated tube and centrifuged immediately. The separated plasma was stored at $-80^{\circ} \mathrm{C}$ until the assays were performed. Plasma $17 \beta$-oestradiol concentration was measured by RIA (DPC estradiol kit; Mitsubishi Chemical Medience Co., Tokyo, Japan). All assays were performed using the same assay kit. The intra-assay CV for $88.3 \mathrm{pg} / \mathrm{ml}$ oestradiol was $6 \cdot 38 \%$.

Data are shown as means with their standard errors. A two-way ANOVA with repeated measures (one within and one between factor) was performed to determine the effects of oestradiol replacement (between factor) and the lightdark phase (within factor) on food intake. A two-way ANOVA (two between factors) was performed to determine the effects of oestradiol replacement and the light-dark phase on the number of c-Fos-IR nuclei in the SCN. Differences between data of specific comparison were determined using the Tukey-Kramer post boc test or Student's $t$ test. Values of $P<0.05$ were considered statistically significant.

\section{Results}

Plasma 17ß-oestradiol concentration was 219.3 (sEM $39.9) \mathrm{pg} / \mathrm{ml}$ in the E2 group and 8.6 (SEM 1.6$) \mathrm{pg} / \mathrm{ml}$ in the Veh group, showing a significant difference between the two groups $(P<0 \cdot 001)$.

The increase in body weight on the 14 th day after the OVX was less in the E2 group (11.4 (SEM 1.4) g) than in the Veh group $(39.6(\operatorname{sem} 3 \cdot 1) \mathrm{g})(P<0.001)$. The mean food intake over the week was less in the E2 group than in the Veh group for both the light and dark phases. However, the mean food intake relative to body weight was significantly less in the E2 group compared with the Veh group only during the light phase (Fig. 1). In addition, the fraction of the total food intake that occurred during the light phase was significantly less in the E2 group (21.7 (SEM 2.2) \%) than in the Veh group (28.5 (SEM 2.3) \%).

The number of c-Fos-IR nuclei in the SCN as a whole showed a clear diurnal rhythm (Fig. 2) and was significantly greater during the light phase compared with the dark phase in both the E2 and Veh groups (Fig. 3(a)). The number of c-Fos-IR nuclei during the light phase was significantly greater in the E2 group compared with the Veh group. However, during the dark phase, there was no difference in
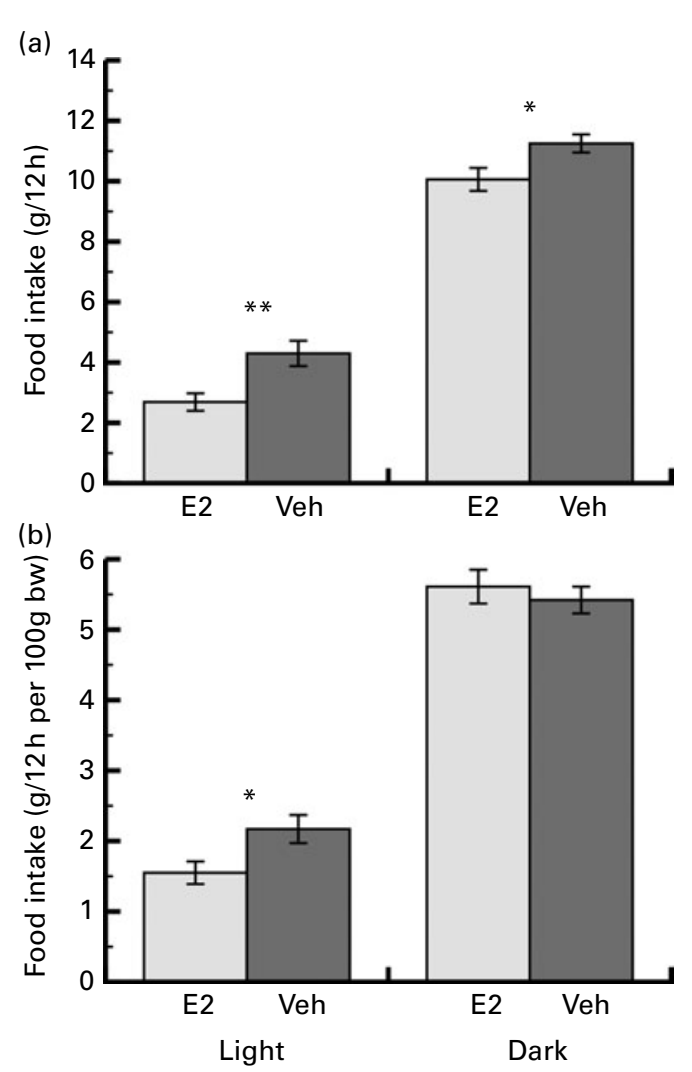

Fig. 1. Mean (a) food intake and (b) food intake relative to body weight (bw) during the light and dark phases over a week in the oestradiol-treated group (E2 group, $n$ 6) and in the vehicle-treated group (Veh group, $n$ 6) of ovariectomised rats. Values are means with their standard errors. Mean values were significantly different between the E2 and Veh groups: ${ }^{*} P<0.05$, ${ }^{\star \star} P<0.01$, respectively.

the number of c-Fos-IR nuclei between the E2 and Veh groups (Fig. 3(a)).

In the vl-SCN, the number of c-Fos-IR nuclei in the E2 group during the light phase was significantly greater than that in the Veh group, whereas this number was similar between these groups during the dark phase. The number of c-Fos-expressed cells in the vl-SCN was significantly greater during the light phase than during the dark phase in the E2 group, but there was no difference shown in this number between the light and dark phases for the Veh group (Fig. 3(b)).

In the $\mathrm{dm}-\mathrm{SCN}$, the number of c-Fos-IR cells during the light phase was significantly greater than during the dark phase in both the groups. However, there was no difference shown in the number of c-Fos-IR cells in the dm-SCN between the E2 and Veh groups during the light or the dark phase (Fig. 3(c)).

The number of c-Fos ir cells in the i-SCN was 244 (SEM 73) for the E2 group and 216 (SEM 75) for the Veh group during the light phase, and the number of these cells was 121 (SEM 46) for the E2 group and 134 (SEM 25) for the Veh group during the dark phase. There was no difference between the E2 and Veh groups for each phase or between the light and dark phases for each group. 

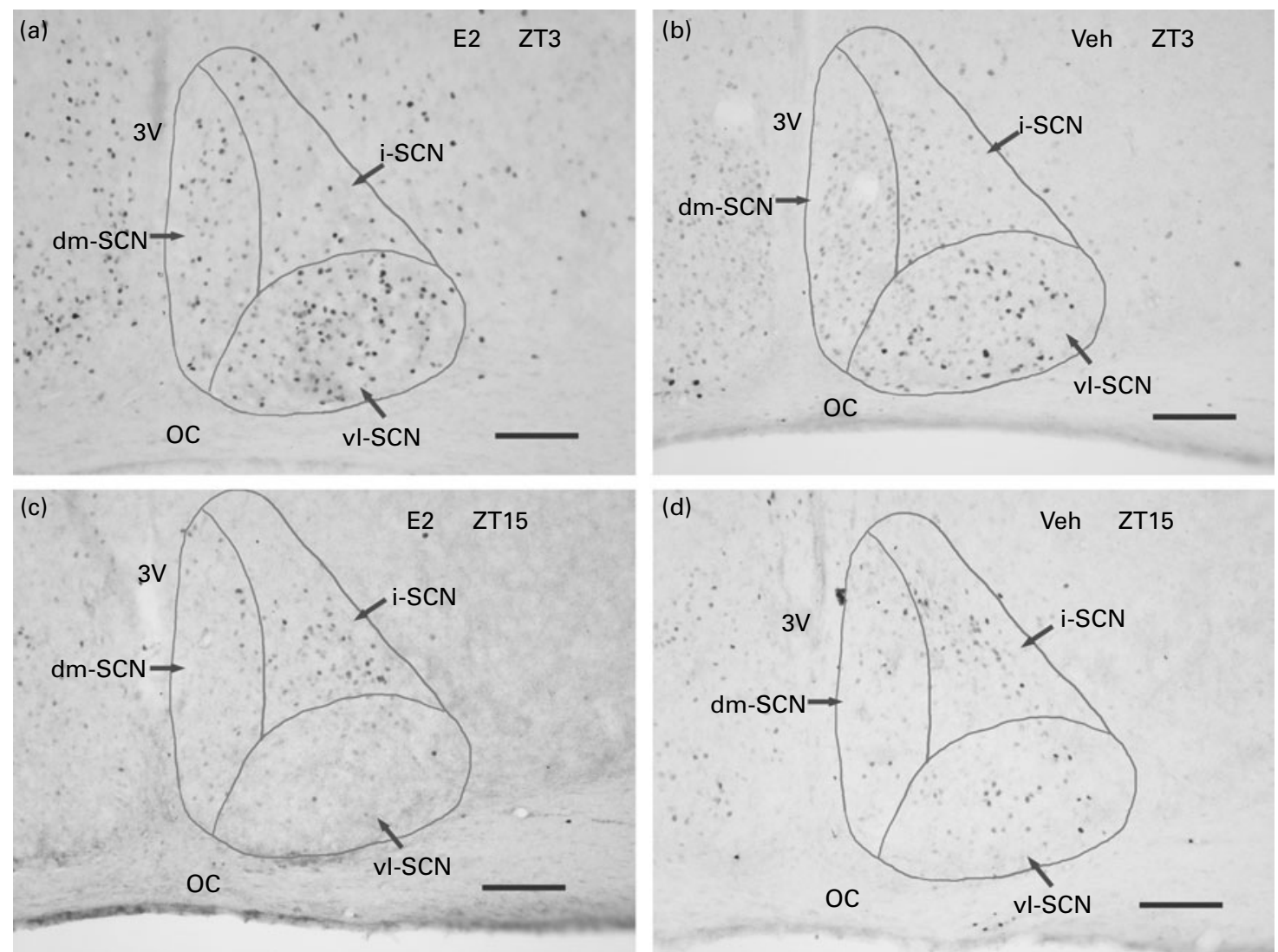

Fig. 2. Representative light microscope photographs of c-Fos-immunoreactive nuclei in the suprachiasmatic nucleus (SCN) in the (a and c) oestradiol-replaced group (E2 group) and in the ( $b$ and d) vehicle-replaced group (Veh group) of ovariectomised rats during $(a$ and $b$ ) the light (Zeitgeber time (ZT)3, 10.00 hours) and (c and d) dark (ZT15, 22.00 hours) phases. vl-SCN, ventrolateral SCN; dm-SCN, dorsomedial SCN; i-SCN, intermediary SCN; OC, optic chiasm; 3V, third ventricle. Scale bars $=100 \mu \mathrm{m}$.

\section{Discussion}

The results obtained by the present study clearly demonstrated that the number of c-Fos-IR cells in the SCN was greater in E2-replaced rats than in E2-deficit rats, specifically during the early light phase 2 weeks after the onset of replacement. The present study is the first to demonstrate that chronic oestrogen replacement tonically increases neuronal activity of the SCN, specifically during the early light phase. The effect of chronic E2 replacement mainly affected vl-SCN activity during the light phase. We also confirmed that chronic oestradiol replacement attenuated food intake during the light phase $^{(7)}$. These results suggest that the increase in vl-SCN activity that occurs during the light phase is possibly involved in the efferent mechanism for the light phase-specific attenuation of food intake by oestrogen replacement.

The SCN plays a critical role in generating the daily variation in food intake ${ }^{(3)}$. Bilateral lesions of the SCN abolish the daily variation in food intake in rats ${ }^{(10)}$, and the temporal signal generated in the SCN is transmitted to both the feeding (lateral hypothalamus) and satiety (ventromedial nucleus of hypothalamus) centres ${ }^{(12,14)}$, suggesting that SCN rhythmicity is necessary for the generation of the feeding rhythm. As reported, the number of $\mathrm{c}-\mathrm{Fos}$ IR-cells in the $\mathrm{SCN}^{(16-20)}$ and the pattern of food intake ${ }^{(7-10)}$ showed clear diurnal rhythms.
The number of c-Fos-IR cells was greater and the amount of food intake was lower during the light phase, compared with the dark phase, suggesting that increased SCN activity is likely to attenuate food intake in rats. In the present study, the number of c-Fos-IR cells in the SCN was significantly greater and the amount of food intake relative to the body weight was significantly lower in the E2 group than in the Veh group during the light phase. However, there was no difference in these values between the E2 and Veh groups during the dark phase. These results strongly suggest that the modification of SCN activity during the light phase by E2 replacement is involved in the light phase-specific attenuation of food intake in the E2 group. However, the present study did not provide data that directly showed the involvement of the SCN.

The SCN can be divided into neuronal subgroups, i.e. $\mathrm{dm}-\mathrm{SCN}$ and vl-SCN parts. The $\mathrm{dm}-\mathrm{SCN}$ contains arginine vasopressin neurons, whereas the vl-SCN mainly contains vasoactive intestinal peptide neurons and receives input directly from the retina and indirectly through the retino-geniculate hypothalamic tract ${ }^{(11,13,28)}$. The peptide contents in the vl-SCN are mainly regulated by photic stimulation, whereas the peptides in the dm-SCN are independent of lighting conditions $^{(11,13,29-31)}$. In the present study, we examined the numbers of c-Fos-IR cells in the subdivisions of the dm-SCN 
(a) Whole SCN

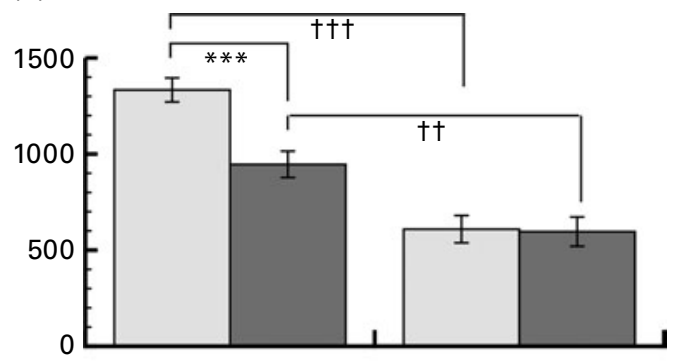

(b) $\mathrm{vl}-\mathrm{SCN}$

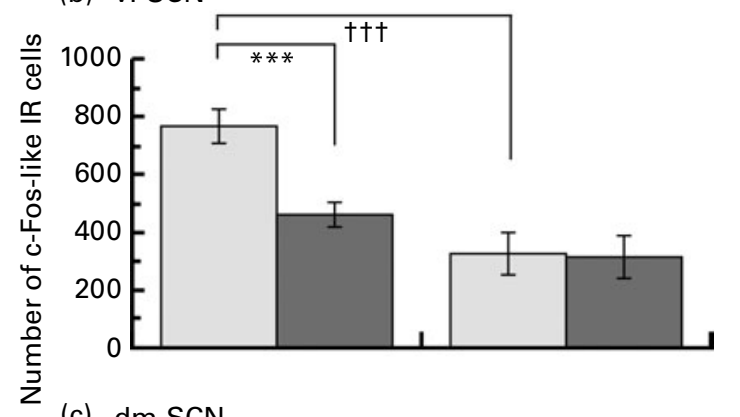

(c) dm-SCN

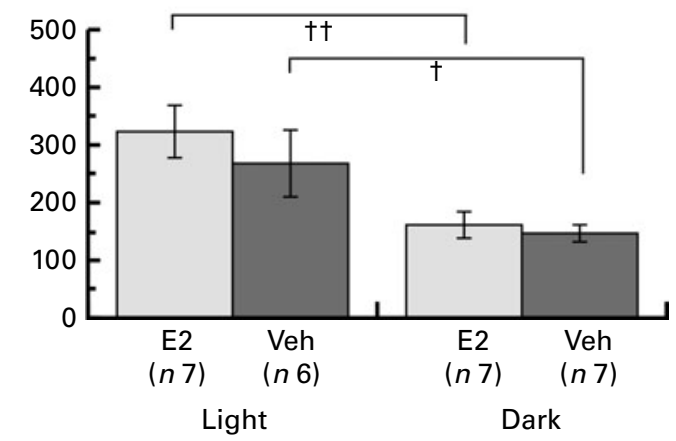

Fig. 3. The numbers of c-Fos-immunoreactive cells in the unilateral (a) whole, (b) dorsomedial (dm)-suprachiasmatic nucleus (SCN)) and (c) ventrolateral (vl-SCN) regions of the SCN in the oestradiol-treated group (E2 group) and in the vehicle-treated group (Veh group) of ovariectomised rats during the light and dark phases. Values are means with their standard errors. The numbers of animals examined are shown in the figure. Mean values were significantly different between the $\mathrm{E} 2$ and Veh groups $\left({ }^{\star \star \star} P<0.005\right)$ Mean values are significantly different between the light and dark phases: $\dagger P<0.05$, †† $P<0.005$, $† \dagger P<0.001$.

and the vl-SCN and in the SCN as a whole. We found that the number of c-Fos-IR cells in the vl-SCN was greater in the E2 group than in the Veh group during the early light phase, but was not greater in the dm-SCN. In addition, the number of c-Fos-IR cells in the vl-SCN did not differ between the light and dark phases for the Veh group. The effect of E2 replacement on SCN activity was specific to the vl-SCN and also specific to the light phase. These results suggest that E2 replacement in OVX rats enhances the response of the vl-SCN itself, augments retina response to light and/or modifies input signals from the oestrogen-sensitive areas, such as the intergeniculate leaflet and dorsal raphe, which project to the $\mathrm{vl}-\mathrm{SCN}^{(18,32)}$. Oestradiol might alter the circadian rhythm outputs for feeding by changing the response of the vl-SCN to light $^{(11,13,18)}$.
Tsukahara ${ }^{(26)}$ examined the role of SCN in controlling luteinising hormone (LH) surges, and reported that the number of c-Fos-IR cells in the vl-SCN subgroup was greater at ZT11-12 in OVX rats receiving an acute E2 injection at ZT8 $2 \mathrm{~d}$ before the c-Fos examination. The data also illustrated that E2 replacement specifically affects the activity of the vl-SCN subgroup. The difference in the timing of the effect of E2 replacement on vl-SCN activity from the data obtained in the present study might be due to the difference in the E2 replacement procedure (chronic or acute) and the timing of the E2 injection.

It has been reported that the SCN receives an input signal of the metabolic status from the ventromedial nucleus of the hypothalamus $^{(3,12)}$ and from the ventromedial arcuate nucleus ${ }^{(33)}$ and that fasting affects SCN activity ${ }^{(19)}$. We could speculate that the modification of SCN activity by oestradiol replacement is a secondary effect that results from the reduction in food intake. Neuropeptide Y (NPY) neurons are located in the ventromedial arcuate nucleus, and oestrogen is known to attenuate the NPY response to hunger stimuli ${ }^{(3)}$ We could also speculate that E2 modifies the metabolic status via these centres, which causes a reduction in food intake and consequently enhances SCN activity. However, the effect of E2 on food intake was light phase-specific, and rats usually do not eat during the early light phase. Liu et $a l .{ }^{(19)}$ reported that fasting reduced the c-Fos expression in the SCN during the light phase but also increased the cFos expression during the dark phase. In addition, restricted feeding schedules entrain clock genes expression in peripheral oscillators but not in the SCN in animals under a lightdark cycle and in constant darkness ${ }^{(34,35)}$. These results suggest that the effect of oestradiol replacement on c-Fos expression in the vl-SCN is not due to the secondary effect of the reduced food intake. It is likely that oestradiol replacement enhances the SCN activity by changing the responsiveness to light, which attenuates food intake specifically during the light phase via efferent signals from the SCN.

The mean food intake over the week was less in the E2 group than in the Veh group for both the light and dark phases. However, the mean food intake relative to body weight was significantly less in the E2 group than in the Veh group only during the light phase, and that the fraction of the total daily food intake that occurred during the light phase was smaller in the E2 group than in the Veh group, suggesting that the attenuation of food intake by chronic E2 replacement is more prominent during the light phase ${ }^{(7)}$. The larger food intake relative to body weight during the light phase contributed, at least in part, to the larger increase in body weight in the Veh group. However, we did not measure the metabolic rate or the locomotor activity in the present study; thus, the relative contribution of food intake and metabolic expenditure to body weight control remains unknown.

Disruption of the circadian rhythm is a factor that can cause obesity $^{(15,21,22,35)}$. In the present study, the daily variations in food intake and in SCN activity were smaller in the Veh group than in the E2 group. The daily variation in food intake in the E2 group was similar to the variation previously 
reported in intact female rats ${ }^{(7)}$. There was no difference in the number of c-Fos-IR cells in the vl-SCN between the light and dark phases in the Veh group. These results suggest that the oestrogen deficit disturbs the circadian rhythm, probably by attenuating the SCN's responsiveness to light, and that the disturbance of the circadian rhythm resulting from the deficit in oestrogen is a possible cause of the increase in body weight and hyperphagia in postmenopausal women. Further studies are needed to elucidate the mechanism of oestrogen deficiency-induced obesity and hyperphagia in relation to the circadian rhythm.

\section{Conclusions}

Our data demonstrated that chronic oestrogen replacement in OVX rats enhances c-Fos expression in the vl-SCN subgroup during the light phase. We also confirmed that oestrogen replacement in OVX rats attenuates food intake, especially during the light phase. Thus, oestrogen may play a role in the enhancement of the SCN's neuronal response to light, which causes an oestrogen-induced attenuation of food intake during the light phase via efferent signals from the SCN.

\section{Acknowledgements}

The present study was supported by a grant-in-aid from the Ministry of Education, Culture, Sports, Science and Technology of Japan, and by a Nara Women's University Intramural Grant for A Takamata's project. We thank Yoko Saito, Rie Kobayashi, Kayo Shibusawa, Yuka Shimizu and Yuka Kobayashi for their technical assistance. A. T. designed the experiment, performed experiments, collected and analysed the data and wrote the paper. K. T. and K. Mi. performed the experiment, collected and analysed the data. K. Mo. provided significant advice on the study design, data analysis and helped to prepare the manuscript. None of the authors had a personal or financial conflict of interest.

\section{References}

1. Butera PC (2010) Estradiol and the control of food intake. Physiol Behav 99, 175-180.

2. Eckel LA (2004) Estradiol: a rhythmic, inhibitory, indirect control of meal size. Physiol Behav 82, 35-41.

3. Kalra SP, Dube MG, Pu S, et al. (1999) Interacting appetiteregulating pathways in the hypothalamic regulation of body weight. Endocrinol Rev 20, 68-100.

4. Svendsen OL, Hassager C \& Christiansen C (1995) Age and menopause-associated variations in body composition and fat distribution in healthy women as measured by dual-energy X-ray absorptiometry. Metabolism 44, 369-373.

5. Asarian L \& Geary N (2002) Cyclic estradiol treatment normalizes body weight and restores physiological patterns of spontaneous feeding and sexual receptivity in ovariectomized rats. Horm Behav 42, 461-471.

6. Santollo J \& Eckel LA (2008) Estradiol decreases the orexigenic effect of neuropeptide $\mathrm{Y}$, but not agouti-related protein, in ovariectomized rats. Behav Brain Res 191, 173-177.
7. Varma M, Chai JK, Meguid MM, et al. (1999) Effect of estradiol and progesterone on daily rhythm in food intake and feeding patterns in Fischer rats. Physiol Behav 68, 99-107.

8. Laviano A, Meguid MM, Gleason JR, et al. (1996) Comparison of long-term feeding pattern between male and female Fischer 344 rats: influence of estrous cycle. Am J Physiol 270, R413-R419.

9. Liu M, Shen L, Liu Y, et al. (2004) Diurnal rhythm of apolipoprotein A-IV in rat hypothalamus and its relation to food intake and corticosterone. Endocrinology 145, 3232-3238.

10. Nagai K, Nishio T, Nakagawa H, et al. (1978) Effect of bilateral lesions of the suprachiasmatic nuclei on the circadian rhythm of food-intake. Brain Res 142, 384-389.

11. Golombek DA \& Rosenstein RE (2010) Physiology of circadian entrainment. Physiol Rev 90, 1063-1102.

12. Challet E (2010) Interactions between light, mealtime and calorie restriction to control daily timing in mammals. J Comp Physiol B 180, 631-644.

13. Ibata Y, Okamura H, Tanaka M, et al. (1999) Functional morphology of the suprachiasmatic nucleus. Front Neuroendocrinol 20, 241-268.

14. Nakagawa H \& Okumura N (2010) Coordinated regulation of circadian rhythms and homeostasis by the suprachiasmatic nucleus. Proc Jpn Acad Ser B Phys Biol Sci 86, 391-409.

15. Rüger $M$ \& Scheer FA (2009) Effects of circadian disruption on the cardiometabolic system. Rev Endocr Metab Disord 10, 245-260.

16. Beaulé C, Barry-Shaw J \& Amir S (2004) Fos expression in the suprachiasmatic nucleus during photic entrainment of circadian rhythms in retinally damaged rats. $J$ Mol Neurosci 22, 223-230.

17. Jác M, Sumová A \& Illnerová H (2000) c-Fos rhythm in subdivisions of the rat suprachiasmatic nucleus under artificial and natural photoperiods. Am J Physiol Regul Integr Comp Physiol 279, R2270-R2276.

18. Karatsoreos IN \& Silver R (2007) The neuroendocrinology of the suprachiasmatic nucleus as a conductor of body time in mammals. Endocrinology 148, 5640-5647.

19. Liu S, Chen XM, Yoda T, et al. (2002) Involvement of the suprachiasmatic nucleus in body temperature modulation by food deprivation in rats. Brain Res 929, 26-36.

20. Peterfi Z, Churchill L, Hajdu I, et al. (2004) Fos-immunoreactivity in the hypothalamus: dependency on the diurnal rhythm, sleep, gender, and estrogen. Neuroscience 124, 695-707.

21. Turek FW, Joshu C, Kohsaka A, et al. (2005) Obesity and metabolic syndrome in circadian Clock mutant mice. Science 308, 1043-1045.

22. Yang S, Liu A, Weidenhammer A, et al. (2009) The role of mPer2 clock gene in glucocorticoid and feeding rhythms. Endocrinology 150, 2153-2160.

23. Vida B, Hrabovszky E, Kalamatianos T, et al. (2008) Oestrogen receptor alpha and beta immunoreactive cells in the suprachiasmatic nucleus of mice: distribution, sex differences and regulation by gonadal hormones. J Neuroendocrinol $\mathbf{2 0}$, $1270-1277$.

24. Fatehi M \& Fatehi-Hassanabad Z (2008) Effects of 17betaestradiol on neuronal cell excitability and neurotransmission in the suprachiasmatic nucleus of rat. Neuropsychopharmacology 33, 1354-1364.

25. Abizaid A, Mezei G \& Horvath TL (2004) Estradiol enhances light-induced expression of transcription factors in the SCN. Brain Res 1010, 35-44.

26. Tsukahara S (2006) Increased Fos immunoreactivity in suprachiasmatic nucleus before luteinizing hormone surge in 
estrogen-treated ovariectomized female rats. Neuroendocrinology 83, 303-312.

27. Paxinos G \& Watson C (2005) The Rat Brain in Stereotaxic Coodinates, 5th ed. San Diego: Academic Press.

28. Aronin N, Sagar SM, Sharp FR, et al. (1990) Light regulates expression of a Fos-related protein in rat suprachiasmatic nuclei. Proc Natl Acad Sci U S A 87, 5959-5962.

29. Mahoney M, Ramanathan C, Hagenauer M, et al. (2009) Daily rhythms and sex differences in vasoactive intestinal polypeptide, VIPR2 receptor and arginine vasopressin mRNA in the suprachiasmatic nucleus of a diurnal rodent, Arvicanthis niloticus. Eur J Neurosci 30, 1537-1543.

30. Shinohara K, Tominaga K, Isobe Y, et al. (1993) Photic regulation of peptides located in the ventrolateral subdivision of the suprachiasmatic nucleus of the rat: daily variations of vasoactive intestinal polypeptide, gastrin-releasing peptide, and neuropeptide Y. J Neurosci 13, 793-800.
31. Sumová A \& Illnerová H (2005) Effect of photic stimuli disturbing overt circadian rhythms on the dorsomedial and ventrolateral SCN rhythmicity. Brain Res 1048, 161-169.

32. Abizaid A, Mezei G, Thanarajasingam G, et al. (2005) Estrogen enhances light-induced activation of dorsal raphe serotonergic neurons. Eur J Neurosci 21, 1536-1546.

33. Yi CX, van der Villet J, Dai J, et al. (2006) Ventromedial arcuate nucleus communicates peripheral metabolic information to the suprachiasmatic nucleus. Endocrinology 147, 283-294.

34. Stokkan KA, Yamazaki S, Tei H, et al. (2001) Entrainment of the circadian clock in the liver by feeding. Science $\mathbf{2 9 1}$, 90-93.

35. Damiola F, Le Minh N, Preitner N, et al. (2000) Restricted feeding uncouples circadian oscillators in peripheral tissues from the central pacemaker in the suprachiasmatic nucleus. Gene Dev 14, 2950-2961. 